\title{
A Comparative Study of Active and Passive Adverse Drug Reaction Reporting Systems in Terms of False Reporting Rate
}

\author{
Alka Bansal, ${ }^{1}$ Ashish Agrawal, ${ }^{2}$ Lokendra Sharma, ${ }^{1}$ Smita Jain ${ }^{3}$
}

\begin{abstract}
Background: World Health Organisation Uppsala Monitoring Centre (WHO-UMC) was set up in 1968 to collect Adverse Drug Reactions (ADRs) periodically for all drugs across the globe. It identifies two main approaches to pharmacovigilance: active (intensive) and passive (spontaneous). However, very few studies are available to compare these two methods of adverse drug reaction reporting.

Methods: A prospective observational study was done on 303 newly diagnosed patients with tuberculosis receiving directly observed therapy short-course (DOTS) in the Sawai Man Singh (SMS) Hospital, Jaipur between 1 January 2019 and 31 December 2019. They were randomly divided into groups A (150 patients) and B (153 patients). Group A patients were followed actively at fixed intervals of time for ADRs till next six months through electronic conversation or personal interview. Group B patients were required to report spontaneously for any ADRs to pharmacovigilance centre. After data collection causality assessment was done using the WHO-UMC scale to identify false reporting and finally results were analysed statistically by means of the t-test using Minitab 14 software Pennsylvania, USA.

Results: 153 ADRs were reported in active and 39 in passive group. Hence the yield of ADR was four times more in active method. After causality assessment, 31 in group A and 12 in group B were found to be falsely related (unlikely) to antitubercular drugs. Two sample t-test revealed active method reported more false ADR ( $p$ $=0.005$ ).

Conclusion: Although active method of surveillance identifies more ADRs than passive method, it is also more prone to false reporting. Hence its benefits should be weighed against its cost before adopting it for countries with limited resources.
\end{abstract}

Key words: Adverse drug reaction; Adverse drug reaction reporting systems; Tuberculosis; Pharmacovigilance.
(1) Department of Pharmacology, SMS Medical College \& Hospital, Jaipur, Rajasthan, India.

(2) Consultant Paediatrician, Jaipur, Rajasthan, India

(3) Department of Mathematics and Statistics, JECRC University, Jaipur, Rajasthan, India.

Correspondence:

ASHISH AGRAWAL

M: +919829044553

E: drashishagrawal73@gmail.com

\section{ARTICLE INFO}

Received: 27 October 2020 Revision received: 13 November 2020 Accepted: 13 November 2020

\section{Introduction}

Adverse Drug Reactions (ADRs) are inevitable part of drug administration. They account for $5 \%$ of all hospital admissions, occur in 10-20 \% of hospitalised patients and are the fourth leading cause of death. ${ }^{1}$ To collect and analyse the ADRs of all drugs across the world, a pharmacovigilance programme (also known as drug safety monitoring abbrevi- ated as DSM or drug surveillance) was started in $1968 .^{2}$ Pharmacovigilance is defined by the World Health Organisation (WHO) as "the science and activities relating to the detection, assessment, understanding and prevention of adverse effects or any other drug-related problem." ${ }^{3}$ 
WHO identifies two main approaches to pharmacovigilance: passive (spontaneous) surveillance and active (intensive) surveillance. ${ }^{4}$ Most of the countries including India usually follow the passive (spontaneous) method of reporting ADR, though, some developed nations like Australia, The Netherlands, Cambodia have well organised active system for drug safety monitoring also using the claims, electronic health records, pharmacy benefits manager, registry, medical records, practitioner-generated data and questionnaire based e-tools. ${ }^{5-7}$ In spontaneous method, ADR reporting is done by health care workers or patients themselves to the pharmacovigilance centre (also known as ADR Monitoring Centre). This method covers a larger population and is useful for hypothesis generation, signal detection and for rare diseases but is often targeted for its under-reporting, the Weber effect (maximum ADRs are reported in first two years of launch of a medicine and then keeps on decreasing). ${ }^{8,9,10}$

On the other hand, in active surveillance method, investigator does not wait for the patients or healthcare facility itself to report ADRs. Rather, they themselves take the initiative to find them in a pre-organised manner. Active surveillance is promoted for early detection of ADRs and signals. ${ }^{11,12}$ Government of India has also initiated active DSM in 2015 for second line and newer drugs used in multi drug resistant (MDR) and extremely drug resistant (XDR) cases of tuberculosis (TB) but there are still negligible data regarding comparison of these two methods of ADR surveillance in terms of actual yield and false reporting. ${ }^{13}$

The objective of the present research was to compare active and passive ADR reporting methods with special focus on false reporting rate in category I tubercular patients. TB was selected owing to high incidence of ADRs due to antituberculosis treatment (ATT) and importance of the disease to the country.

\section{Methods}

It was a prospective, observational type of study conducted at Directly Observed Therapy Shortcourse (DOTS) centre and ADR Monitoring centre (AMC), also known as pharmacovigilance centre, attached to the Sawai Man Singh (SMS) Hospital, Jaipur from 1 January 2019 to 31 December 2019.
A total of 303 category I TB patients who were visiting DOTS centre for the first time for treatment of TB were enrolled into the study after their informed consent. Category I TB comprises newly diagnosed case of TB susceptible to first line antitubercular drugs, ie isoniazid, rifampicin, pyrazinamide, ethambutol. Category II TB patients, those on immunosuppressants, pregnant and lactating women were not included in the study. A total of 303 enrolled patients were randomly divided into two groups A (150) and B (153). If anyone proved to be resistant to any of the above four drugs during the study later on, he/she was excluded and a fresh recruitment was done untill the sample size of 150 in each group was achieved.

Group A (active surveillance) patients were monitored actively for adverse drug reactions. For it, they were interviewed personally through faceto-face meetings or by phone at pre-decided intervals of time (on days $3,7,15,30,90,180$ ) using a pre-validated structured questionnaire. WHO-endorsed ADR forms were then filled up using the collected ADR information.

Group B (passive surveillance) patients registered for receiving ATT were asked to report any adverse reactions either by themselves or through healthcare workers (HCW) to pharmacovigilance centre of the institute. For self-reporting a tollfree phone number was shared with them and a drop box was placed in DOTS centre.

The recruitment period was six months and data were collected for the next six months after ATT had been started in each patient, as ATT is given for six months in category I TB patients as per National Guidelines. The information collected was then analysed statistically by Chi-squared test and two sample t-test using the software Minitab 14 Pennsylvania, USA.

Institutional Ethical clearance was obtained via letter No 4123/MC/EC/2018 dated 9 October 2018.

Causality assessment and identification of false reporting

With the advancement in technology and growing sources of ADR reporting (even social media like Twitter, Facebook), a number of methods and algorithms for filtering entities to optimise ADR identification have been developed. ${ }^{14}$ But as this study was constrained to single institute with 
limited ADRs, so WHO-UMC scale for causality assessment was followed for false reporting also. According to Varallo et al also, it is the most consistent way for use in hospitals, since it allows evaluating the quality of the report. ${ }^{15}$

WHO-UMC system is basically a combined assessment taking into account the clinico-pharmacological aspects of the case, history and the quality of the documentation of the ADR. There are six WHO-UMC causality categories - certain, probable/likely, possible, unlikely, conditional/unclassified and unassessable/unclassifiable. Since by far the most frequent categories in case reports are 'possible' and 'probable', the usual approach is to choose one of these categories (depending on the impression of the assessor) and to test if the various criteria fit with the content of the case report for ADR. To see if that category is the right one or if it does again not seem to fit, the next adjacent term is tried. Those that fell into the category 'unlikely' were considered as falsely attributed to drug(s) being used. ${ }^{16}$

\section{Results}

Characteristics of the patients included in active and passive study groups are shown in Table1.

Chi-squared test applied for the characteristics between two groups gave $p$ value of 0.990 showing no significant difference between the study
Table 1: Characteristics of the patients in active and passive study groups

\begin{tabular}{lcc}
\hline Characteristics & Active (150) & Passive (153) \\
\hline Mean age (years) & 29 & 31 \\
\hdashline Male & 96 & 91 \\
\hdashline Female & 54 & 62 \\
\hdashline Pulmonary TB & 126 & 128 \\
\hline Extrapulmonary TB & 24 & 25 \\
\hline Education - high level and above & 113 & 101 \\
\hline Associated comorbidities & & \\
\hline HIV & 3 & 3 \\
\hline Diabetes mellitus & 10 & 10 \\
\hline Addiction - smoking/alcoholism & 16 & 16 \\
\hline
\end{tabular}

groups.

A total of 192 ADRs were reported from 303 patients in this study. When the ADRs collected by

Table 2: Distribution of false positive ADRs in active and passive methods

\begin{tabular}{lcc}
\hline ADR & Active (150) & Passive (153) \\
\hline Nausea, vomiting & 2 & 2 \\
\hline Gastrointestinal upset & 4 & 2 \\
\hline Abdominal pain & 2 & 1 \\
\hline Jaundice & 1 & 0 \\
\hline Skin rash & 3 & 1 \\
\hline Pruritus & 4 & 1 \\
\hline Flushing & 2 & 1 \\
\hline Paraesthesia & 2 & 0 \\
\hline Numbness & 0 & 0 \\
\hline Visual toxicity & 2 & 0 \\
\hline Mental disturbances & 3 & 2 \\
\hline Loss of diabetes control & 1 & - \\
\hline Arthritis & 1 & 0 \\
\hline Decreased urine output & 0 & 0 \\
\hline Others - fever, shock, & 4 & 2 \\
\hline acne, vertigo, dyspnoea & & 2 \\
\hline
\end{tabular}

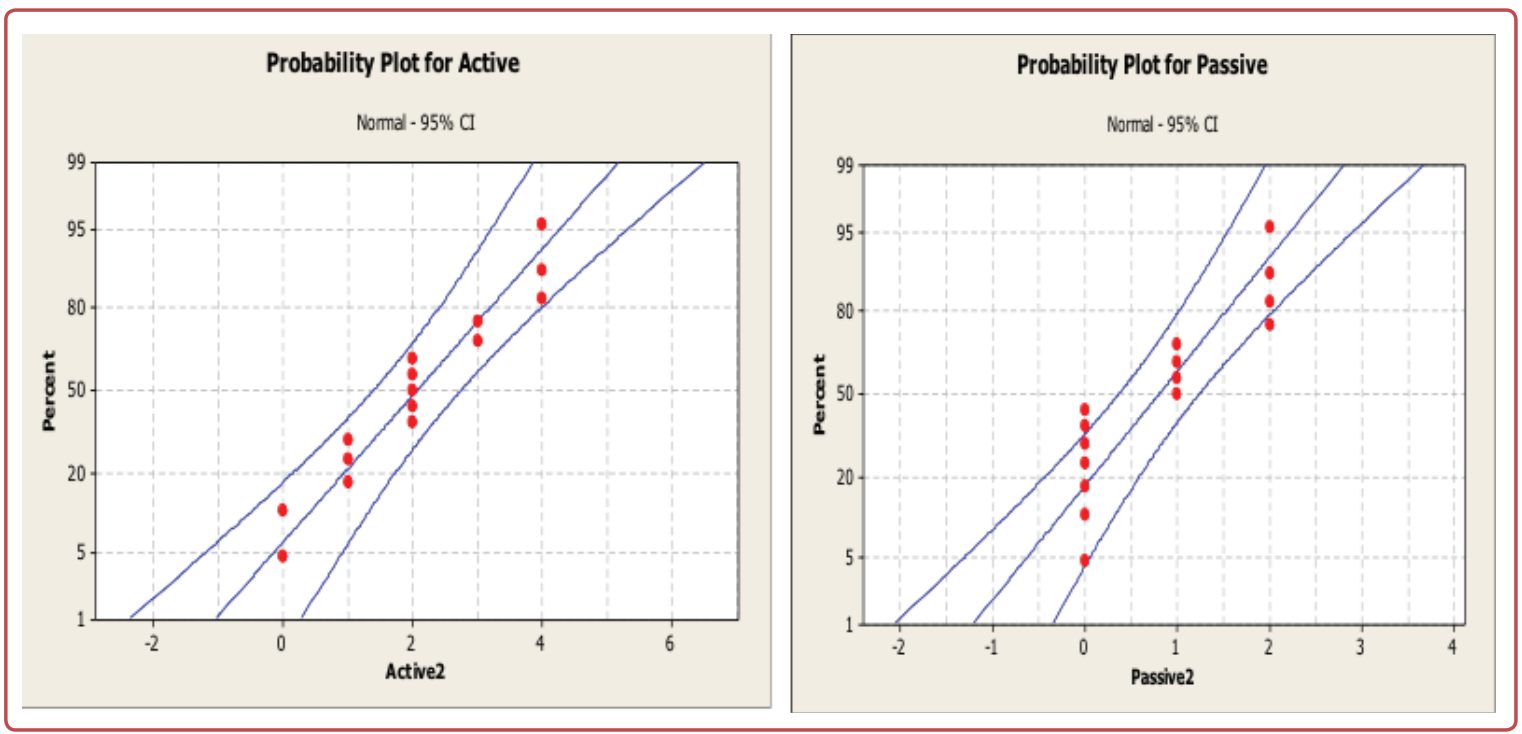

Figure 1: Normality check of the data distribution for active and passive surveillance 
active and passive surveillance method separately were compared, it revealed that the ratio of ADR detection was 4:1 in active verses passive ADR surveillance group. By active method, a total of 153 ADRs were reported and 39 ADRs were collected by passive reporting. On causality assessment of the reports, it was seen that out of them 31 and 12 were falsely attributed to ATT by active and passive methods, respectively (Table 2). These falsely reported ADRs were either not in timeline with intake of drug or other diseases/ drugs provided plausible explanations.

\section{Statistical analysis and interpretation}

Initially normality of the data distribution was checked by probability plots before applying the t-test. As almost all the points lied within the $95 \%$ confidence interval, it was inferred that the study population in both the groups was following the normal distribution (Figure 1).

Then the two-sample t-test was applied for statistical comparison of false reporting rate between the active and passive methods (Table 3). To apply the t-test it was assumed that null hypothesis $\left(\mathrm{H}_{0}\right)$ was true and hence there was no difference between them.

Table 3: Two-sample t-test and confidence interval (Cl) for false ADRs in active and passive method

\begin{tabular}{lcccc}
\hline & N & Mean & SD & SE \\
\hline Active & 15 & 2.07 & 1.33 & 0.34 \\
\hline Passive & 15 & 0.80 & 0.86 & 0.22 \\
\hline
\end{tabular}

$95 \% \mathrm{Cl}$ for difference: $(0.41,2.11) ; t=3.09 ; p=0.005$;

$N=$ number of variables, $S D=$ standard deviation; $S E=$ standard error of means

Since this p value was less than 0.05 (level of significance in the study) so the null hypothesis was rejected and it was concluded that there was a significant difference of false reporting rate between the methods. As the mean, standard deviation, standard error of mean (SE) mean values were higher for active method, it means high incidence of false reporting in active in comparison to passive method.

\section{Discussion}

Isoniazid, rifampicin, pyrazinamide, ethambutol are the first line anti-TB drugs given in all category I TB patients and some category II TB patients depending on susceptibility. Anti-TB drugs are documented to have high incidences of ADRs, next to chemotherapy and antiretroviral drugs. ${ }^{17}$ The most common ADRs induced by first-line anti-TB drugs are hepatotoxicity, gastrointestinal disorders, allergic reactions, arthralgias, neurological disorders etc. In this study, active surveillance and spontaneous reporting for ADR contributed $80 \%$ and $20 \%$ of total ADRs, respectively. Similar study by Tandon et al reported $66.13 \%$ versus $33.86 \%$ of the total ADRs. ${ }^{18}$ Many other studies have also shown that that active surveillance programs detected additional ADRs compared to those of spontaneous reporting programs. They noted not only causative drugs, clinical features also varied in two methods. ${ }^{18,19,20}$

In the present study, after causality assessment, $22.3 \%$ of ADRs were found to be falsely attributed to ATT with $72.1 \%$ and $27.9 \%$ from active and passive reporting methods, respectively. Statistical analysis confirmed that active method is prone to false reporting of ADR ( $p=0.005)$. These findings are in concordance to the study by Berry et al that for level of specificity, the decreasing order for the three ADR reporting methods was voluntary reports (passive), pharmacist screening of medication orders and pharmacist screening of laboratory reports (active); with the differences among all three methods being significant. ${ }^{21}$ Tandon et al also raised the concern of false reporting if it is made mandatory for HCW and thus compromising the quality of reports..$^{18}$

The main purpose of the study presented in this paper was to investigate the potential of a surveillance system for yield and false reporting and not to evaluate the drug reactions reported. Presented findings are akin to those by Lynn et al who also found that although two systems are clearly different, they could complement each other. ${ }^{22}$ If an active reporting system is to be used in routine for ADR collection, it will be necessary to improve ADR ascertainment and decrease false reporting.

\section{Strengths of the study}

This is the first data-based study to compare active and passive methods in terms of yield and false reporting. 


\section{Limitations of the study}

The study was restricted to a particular disease in a single institution.

\section{Conclusion}

Active surveillance for ADRs definitely increases the yield in comparison to passive spontaneous reporting but at the same time chances of false reporting are also significantly higher in it.

\section{Acknowledgements}

The authors express their gratitude to the staff of DOTS centre for their help in recruiting the study participants. They are also thankful to the patients who participated in the study.

\section{Conflict of interest}

None.

\section{References}

1. Syed Hussain F, Sathyanarayanan V, Jamuna Rani R. Analysis of adverse drug reactions encountered in a tertiary care hospital: a cross sectional study. Int J Basic Clin Pharmacol 2018 Jun;7(6):1164-8.

2. Lindquist M, Edwards I. The WHO Programme for International Drug Monitoring, its database, and the technical support of the Uppsala Monitoring Center. J Rheumatol 2001;28:1180-7.

3. World Health Organization. Essential medicines and health products, pharmacovigilance [Cited on 2020 oct 26]. Availble from: https://www.who.int/medicines/areas/quality_safety/safety_efficacy/pharmvigi/en/.

4. Weaver J, Willy M, Avigan M. Informatic tools and approaches in postmarketing pharmacovigilance used by FDA. AAPS J 2008 Mar 1;10(1):35-41.

5. Huang YL, Moon J, Segal JB. A comparison of active adverse event surveillance systems worldwide. Drug Saf 2014 Aug 1;37(8):581-96.

6. Baron S, Goutard F, Nguon K, Tarantola A. Use of a text message-based pharmacovigilance tool in Cambodia: pilot study. J Med Internet Res 2013;15(4):e68. doi: 10.2196/jmir.2477.
7. Suke SG, Kosta P, Negi H. Role of pharmacovigilance in India: an overview. Online J Public Health Inform 2015 Jul 1;7(2):e223. doi: 10.5210/ojphi.v7i2.5595.

8. Matsuda S, Aoki K, Kawamata T, Kimotsuki T, Kobayashi $\mathrm{T}$, Kuriki $\mathrm{H}$, et al. Bias in spontaneous reporting of adverse drug reactions in Japan. PLoS One 2015 May 1;10(5):e0126413. doi: 10.1371/journal.pone.0126413.

9. Norén GN, Edwards IR. Modern methods of pharmacovigilance: detecting adverse effects of drugs. Clin Med (Lond) 2009 Oct;9(5):486-9.

10. Inácio $\mathrm{P}$, Cavaco $\mathrm{A}$, Airaksinen $\mathrm{M}$. The value of patient reporting to the pharmacovigilance system: a systematic review. Br J Clin Pharmacol 2017 Feb;83(2):227-46.

11. Gliklich RE, Dreyer NA, Leavy MB, eds. Adverse event detection, processing, and reporting. In: Registries for evaluating patient outcomes: a user's guide [Internet]. 3rd edition. Rockville (MD): Agency for Healthcare Research and Quality; 2014.

12. Wu T, Gao CC, Lin JS, Zha JL. Active monitoring of adverse drug reactions with neural network technology. Chin Med J (Engl) 2017 Jun 20;130(12):1498-501.

13. World Health Organisation. Active TB drug-safety monitoring and management (aDSM ) [cited on 2020 Oct 26] Available from: https://www.who.int/tb/areas-of-work/drug-resistant-tb/treatment/adsm_factsheet_2018.pdf

14. Abdellaoui R, Schück S, Texier N, Burgun A. Filtering entities to optimize identification of adverse drug reaction from social media: how can the number of words between entities in the messages help? JMIR Public Health Surveill 2017 Jun 22;3(2):e36. doi: 10.2196/publichealth.6577.

15. Varallo FR, Planeta CS, Herdeiro MT, Mastroianni PC. Imputation of adverse drug reactions: Causality assessment in hospitals. PloS One 2017 Feb 6;12(2):e0171470. doi: 10.1371/journal.pone.0171470.

16. Srinivasan R, Ramya G. Adverse drug reaction-causality assessment. Int J Res Pharm Chem 2011;1(3):606-12.

17. Ministry of Health and Family Welfare. India TB Report 2020. [cited on 2020 Oct 26]. Available from: https://tbcindia.gov.in/showfile.php?lid=3538.

18. Tandon VR, Mahajan V, Khajuria V, Gillani Z. Under-reporting of adverse drug reactions: a challenge for pharmacovigilance in India. Indian J Pharmacol 2015;47(1):65-71.

19. Jha AK, Kuperman GJ, Teich JM, Leape L, Shea B, Rittenberg E, et al. Identifying adverse drug events: development of a computer-based monitor and comparison with chart review and stimulated voluntary report. J Am Med Inform Assoc1998 May 1;5(3):305-14.

20. Yun IS, Koo MJ, Park EH, Kim SE, Lee JH, Park JW, et al. A comparison of active surveillance programs including a spontaneous reporting model for phamacovigilance of adverse drug events in a hospital. Korean J Intern Med 2012 Dec;27(4):443-50.

21. Berry LL, Segal R, Sherrin TP, Fudge KA. Sensitivity and specificity of three methods of detecting adverse drug reactions. Am J Hosp Pharm 1988 Jul 1;45(7):1534-9.

22. Lynn RM, Riding K, McIntosh N. The use of electronic reporting to aid surveillance of ADRs in children: a proof of concept study. Arch Dis Child 2010 Apr 1;95(4):262-5. 